\title{
Editorial: Movement Disorders and Sleep - Underlying Mechanisms, Clinical Aspects and Treatment
}

\author{
Cristian Falup-Pecurariu ${ }^{1 *}$, Nataliya Titova ${ }^{2}$ and K. Ray Chaudhuri ${ }^{3}$ \\ 1 Department of Neurology, Faculty of Medicine, County Emergency Clinic Hospital, Transilvania University Braşov, Braşov, \\ Romania, ${ }^{2}$ Department of Neurology, Neurosurgery and Medical Genetics, Pirogov Russian National Research Medical \\ University, Moscow, Russia, ${ }^{3}$ Parkinson Foundation Centre of Excellence, King's College London, King's College Hospital, \\ London, United Kingdom
}

Keywords: movement disorders, sleep, treatment, mechanisms, assessment

\section{Editorial on the Research Topic}

\section{Movement Disorders and Sleep - Underlying Mechanisms, Clinical Aspects and Treatment}

Nocturnal sleep dysfunction is a key problem in many movement disorders and one of the major non-motor symptoms in Parkinson's disease (PD) the world's fastest growing neurodegenerative disorder with consequences during waking hours at daytime as well as daily functioning (1). In PD for example, sleep dysfunction such as nocturnal waking and daytime somnolence was recognized by James Parkinson himself and continues to be a clinical challenge given the problems arise from the motor problems at night, neurotransmitter driven alterations in sleep architecture as well as drug related effects $(2,3)$. Non-motor endophenotypes of PD have been recognized and serotonergic dysfunction for instance in the raphe and limbic areas could drive aspects of sleep dysfunction in PD and some other related neurodegeneration (4).

In this special edition of the Frontiers in Neurology, sleep dysfunction in Movement Disorders is

\section{OPEN ACCESS}

Edited and reviewed by: Martina Mancini,

Oregon Health \& Science University, United States

*Correspondence: Cristian Falup-Pecurariu crisfp100@yahoo.co.uk

Specialty section:

This article was submitted to Movement Disorders,

a section of the journal

Frontiers in Neurology

Received: 27 July 2019

Accepted: 11 September 2019

Published: 17 October 2019

Citation:

Falup-Pecurariu C, Titova $N$ and Ray

Chaudhuri K (2019) Editorial:

Movement Disorders and Sleep Underlying Mechanisms, Clinical

Aspects and Treatment.

Front. Neurol. 10:1034.

doi: 10.3389/fneur.2019.01034 addressed focusing on possible pathophysiological mechanisms, clinical aspects of recognition, and awareness and treatment. Yousaf et al. provide a narrative review of the various types of molecular, structural, and functional neuroimaging techniques such as magnetic resonance imaging (MRI), functional magnetic resonance imaging (fMRI), diffusion tensor imaging (DTI), single-photon emission computed tomography (SPECT), and positron emission tomography (PET) that have been explored to provide evidence base for assessing structural, functional and neurochemical correlates of sleep disturbances in PD and other movement disorders such as Huntington's disease and Multiple system atrophy. These data might eventually lead to new drug discovery or repurposing of existing molecules to treat aspects of sleep dysfunction, currently a key unmet need.

Excessive daytime sleepiness (EDS) can be a disabling problem in $\mathrm{PD}$, particularly in the more advanced stages although EDS is also recognized as a prodromal feature of PD and present all through the various clinical stages of $\operatorname{PD}(5,6)$. In some the problem may even result in a clinical syndrome resembling narcolepsy without cataplexy with functional consequences such as sudden onset sleep episodes during driving which can be precipitated by some dopaminergic drugs (7). Biomarkers to detect this variant within PD is therefore, important and Ashraf-Ganjouei et al. describe a Diffusion MRI connectometry study in PD patients with EDS vs. those without and report decreased MRI based connectivity in the left and right fornix, left and right inferior longitudinal fasciculus (ILF), left inferior and middle cerebellar peduncles. Sherbaf et al. describe another diffusion MRI connectometry study in RBD as well as depression, a prodromal NMS, in 93 treatment-naïve and non-demented early PD and report that these two non-motor symptoms may be associated with lower connectivity in several white matter tracts with involvement of short association fibers (U-fibers).

Prodromal and pre prodromal stages of PD are emerging as key areas where early treatment initiation may be a priority and REM sleep behavior disorder (RBD) is a clinical biomarker for the 
development of $\alpha$-synucleinopathies as well as a predictor of early cognitive decline (8). Excessive daytime sleepiness (EDS) can also be a prodromal non-motor symptom of PD. Gjerstad et al. address these two key sleep related symptoms in PD and discuss the implications in early stages of $\mathrm{PD}$ as well as the regulators of gene expression and core clock genes such as CLOCK and ARNTL (BMAL1) and the potential association of dopaminergic therapies and circadian genetic markers in PD.

Clinical assessment of sleep dysfunction is crucial for assessing the problem, addressing efficacy of treatment as well as providing validated outcomes for value based healthcare and in several papers, Rodríguez-Blázquez et al., Kurtis et al., and Skorvanek et al. discuss the roles, clinimetrics as well as application potential of clinical scales used to assess sleep dysfunction in PD and other movement disorders. Bhidayasiri et al. report an international study between Thailand, Japan, and India where they report on the utility of a PD Home Safety Questionnaire and discuss implications for adaptations based on a novel concept of concept of Personal (P)-Environmental (E) fit. Restless Legs Syndrome (RLS) often complicate sleep in PD and a link between the two conditions remain viable yet controversial. This issue is discussed by Ferrini-Strambi et al. who argue the case for well-designed systematic and strongly controlled longitudinal studies to clarify the aforesaid relationship.

Wearable sensors and the use of digital technology has become a topic of major research and clinical interest in the

\section{REFERENCES}

1. Ray Chaudhuri K, Titova N. Societal burden and persisting unmet needs of Parkinson's disease. Eur Neurol Rev. (2019) 14:3-9. doi: 10.17925/ENR.2019.14.1.28

2. Parkinson J. An Essay on the Shaking Palsy. 1817. J Neuropsychiatry Clin Neurosci. (2002) 14:223-36; discussion 222. doi: 10.1176/jnp.14.2.223

3. Falup-Pecurariu C, Diaconu Ş. Sleep dysfunction in Parkinson's disease. Int Rev Neurobiol. (2017) 133:719-42. doi: 10.1016/bs.irn.2017.05.033

4. Titova N, Padmakumar C, Lewis SJG, Chaudhuri KR. Parkinson's: a syndrome rather than a disease? I Neural Transm. (2017) 124:907-14. doi: 10.1007/s00702-016-1667-6

5. Chaudhuri, KR, Healy DG, Schapira AHV. Non-motor symptoms of Parkinson's disease: diagnosis and management. Lancet Neurol. (2006) 5:23545. doi: 10.1016/S1474-4422(06)70373-8

6. Berg D, Postuma RB, Adler CH, Bloem BR, Chan P, Dubois B, et al. MDS research criteria for prodromal Parkinson's Disease. Mov Disord. (2015) 30:1600-11. doi: $10.1002 / \mathrm{mds} .26431$ field of movement disorders. Sleep has been an increasing focus of research in this field and Madrid-Navarro et al. provide an interesting cross-sectional study using a wristworn device combined with machine-learning so as to process and detect circadian rhythms of sleep, motor, and autonomic disruption in PD providing an index to multidimensional circadian monitoring.

Advanced therapies in PD have now been shown to have beneficial or occasionally disruptive effects on sleep in PD. Sharma et al. review the role of deep brain stimulation at different anatomical targets which may affect the sleep-wake cycle via multiple factors, including motor symptoms, medication adjustment, and direct modulation of sleep-wake centers in PD.

This edition of the special Research Topic addressing sleep dysfunction in movement disorders therefore, present a series of cutting edge papers with original research data including neuroimaging and other biomarkers as well as reviews spanning clinical aspects of sleep dysfunction in PD and other movement disorders including RLS. We hope that the frontiers in sleep research in movement disorders would be facilitated by work presented in this special edition.

\section{AUTHOR CONTRIBUTIONS}

KR drafted and revised the manuscript for intellectual content. CF-P and NT revised the manuscript for intellectual content.

7. Ylikoski A, Martikainen K, Sarkanen T, Partinen M. Parkinson's disease and narcolepsy-like symptoms. Sleep Med. (2015) 16:540-4. doi: 10.1016/j.sleep.2014.12.010

8. Siderowf A, Lang A. Premotor Parkinson's disease: concepts and definitions. Mov Disord. (2012) 27:608-16. doi: 10.1002/mds. 24954

Conflict of Interest: The authors declare that the research was conducted in the absence of any commercial or financial relationships that could be construed as a potential conflict of interest.

Copyright (c) 2019 Falup-Pecurariu, Titova and Ray Chaudhuri. This is an openaccess article distributed under the terms of the Creative Commons Attribution License (CC BY). The use, distribution or reproduction in other forums is permitted, provided the original author(s) and the copyright owner(s) are credited and that the original publication in this journal is cited, in accordance with accepted academic practice. No use, distribution or reproduction is permitted which does not comply with these terms. 